\title{
Cross sectional echocardiographic identification of hypoplastic left heart syndrome and differentiation from other causes of right ventricular overload
}

\author{
Peter Ludman, Rodney Foale, Neal Alexander, Petros Nihoyannopoulos
}

\begin{abstract}
To identify the echocardiographic features that can be used to distinguish between hypoplastic left heart syndrome and other causes of right ventricular overload in the sick neonate cross sectional echocardiographic studies of 10 neonates with hypoplastic left heart syndrome were analysed and compared with those in 15 neonates with other causes of right ventricular overload and 15 normal controls. Left ventricular and right ventricular cavity dimensions and the shape and size of the mitral valve annulus and aortic root were recorded and presented both as absolute values ( $\mathrm{mm}$ ) and corrected for body surface area $\left(\mathrm{mm} / \mathrm{m}^{2}\right)$. Logistic regression was used to produce a classification rule to estimate the probability of a neonate having hypoplastic left heart syndrome. The diameter of the mitral valve annulus was the single most discriminative variable. There was, however, considerable overlap of all the calculated features between neonates with hypoplastic left heart syndrome and those with other causes of right ventricular overload.
\end{abstract}

The diagnosis of hypoplastic left heart syndrome should not be based on any one single echocardiographic feature but the general appearance of abnormal left heart valves, small cavity dimensions, and the size of the mitral valve annulus.

Cross sectional echocardiography has enhanced the diagnosis of congenital heart disease and in particular of cardiac abnormality in the newborn. ${ }^{1}$ With conventional treatment, hypoplastic left heart syndrome is invariably fatal and must be distinguished early from potentially operable conditions which may have a similar clinical and echocardiographic presentation.

Previous reports identified the echocardiographic features of hypoplastic left heart syndrome: the main one is an abnormally small left ventricular cavity compared with the right ventricle. ${ }^{2-7}$ Though some workers reported that associated cardiac abnormalities aided echocardiographic diagnosis, ${ }^{589}$ the wide range of cardiac lesions in this syndrome ${ }^{10}$ makes it difficult to distinguish between hypoplastic left heart syndrome and other causes of right ventricular dominance.
We sought to describe the range of cardiac abnormalities associated with hypoplastic left heart syndrome and to identify the echocardiographic variables that distinguish the sick neonate with hypoplastic left heart syndrome from infants with other causes of right ventricular overload.

\section{Patients and methods}

Hypoplastic left heart syndrome was diagnosed in 10 neonates referred to the cardiac ultrasound laboratory at the Hammersmith Hospital over a period of five years. In nine the diagnosis was confirmed at necropsy and in one patient at cardiac catheterisation and operation. This patient subsequently died and permission for a necropsy was refused (table 1).

We also studied 15 neonates with other causes of right ventricular overload (table 2) and 15 clinically normal neonates with similar age and body surface area (table 3 ).

\section{METHODS}

Complete echocardiographic studies were performed in all patients according to a well standardised protocol, ${ }^{11}$ particular attention was given to imaging the right heart chambers from multiple views. ${ }^{12}$ We used suprasternal and high parasternal views to image the aortic arch. ${ }^{13}$ We used ATL Mark 100C ultrasound equipment with a 5 and a $7 \mathrm{MHz}$ mechanical transducer or a Toshiba SSH 160A with a $3.75 \mathrm{MHz}$ and a $5 \mathrm{MHz}$ phased array transducer. In all neonates we used appropriate onaxis projections of right and left ventricles from parasternal long axis views to measure the maximum dimension of the left and right ventricular cavities and the thickness of the posterior left ventricular wall and interventricular septum. These measurements were taken at the level of the chordae tendineae (fig 1A). We used cross sectional short axis projections to measure the anteroposterior and lateral left ventricular diameters to assess possible geometric distortion of the left ventricle secondary to right ventricular overload (fig 1B). Aortic root dimensions were measured from the parasternal long axis projection, the maximum diameter being recorded at the sinus, sinotubular junction, and ascending aorta (fig 1C). The size of the mitral valve annulus was assessed from the apical four chamber projection (fig 1D).

All the chamber dimensions and mitral annulus diameter were measured at end diastole; the aortic root and ascending aorta were 
Table 1 Clinical and echocardiographic features of the 10 neonates with hypoplastic left heart syndrome

\begin{tabular}{|c|c|c|c|c|c|c|c|c|c|c|c|c|c|c|}
\hline \multirow[b]{2}{*}{ Patient } & \multirow{2}{*}{$\begin{array}{l}\text { Age at } \\
\text { study } \\
\text { (days) }\end{array}$} & \multirow{2}{*}{$\begin{array}{l}\text { Presenting } \\
\text { symptom }\end{array}$} & \multirow{2}{*}{$\begin{array}{l}\text { Weight } \\
\text { (kg) }\end{array}$} & \multirow{2}{*}{$\begin{array}{l}B S A \\
\left(m^{2}\right)\end{array}$} & \multicolumn{2}{|l|}{$L V$} & \multicolumn{2}{|l|}{$S A X$} & \multicolumn{2}{|l|}{$R V$} & \multicolumn{2}{|c|}{$L V P W$} & \multicolumn{2}{|l|}{ IVS } \\
\hline & & & & & $(m m)$ & $\left(\mathrm{mm} / \mathrm{m}^{2}\right)$ & $(A P)(m m)$ & $(L A T)(\mathrm{mm})$ & $(\mathrm{mm})$ & $\left(\mathrm{mm} / \mathrm{m}^{2}\right)$ & $(\mathrm{mm})$ & $\left(\mathrm{mm} / \mathrm{m}^{2}\right)$ & $(m m)$ & $\left(\mathrm{mm} / \mathrm{m}^{2}\right)$ \\
\hline $\begin{array}{l}1 \\
2 \\
3 \\
4 \\
5 \\
6 \\
7 \\
8 \\
8\end{array}$ & $\begin{array}{l}2 \\
3 \\
5 \\
6 \\
1 \\
9 \\
2 \\
5\end{array}$ & $\begin{array}{l}\text { C, T, F } \\
\text { C, T, F } \\
\text { A, T, F } \\
\text { A, T, F } \\
\text { A, T, F } \\
\text { C, T, F } \\
\text { A, T, F }\end{array}$ & $\begin{array}{l}3.3 \\
3.5 \\
3.0 \\
3.4 \\
2.9 \\
3.0 \\
3.4 \\
2.7\end{array}$ & $\begin{array}{l}0.21 \\
0.22 \\
0.20 \\
0.22 \\
0.21 \\
0.21 \\
0.21 \\
0.18\end{array}$ & $\begin{array}{r}12 \\
11 \\
16 \\
7 \\
11 \\
2 \\
2 \\
8 \\
13\end{array}$ & $\begin{array}{l}56.6 \\
50.0 \\
80.0 \\
32.1 \\
52.9 \\
9.8 \\
38.1 \\
72.2\end{array}$ & $\begin{array}{r}8 \\
10 \\
15 \\
11 \\
13 \\
2 \\
13 \\
10\end{array}$ & $\begin{array}{r}10 \\
12 \\
15 \\
10 \\
11 \\
3 \\
15 \\
15\end{array}$ & $\begin{array}{l}10 \\
19 \\
15 \\
18 \\
16 \\
33 \\
12 \\
16\end{array}$ & $\begin{array}{r}47.2 \\
86.4 \\
75.0 \\
82.6 \\
76.9 \\
161.0 \\
57.1 \\
88.9\end{array}$ & $\begin{array}{l}8 \\
2 \\
5 \\
3 \\
8 \\
2 \\
4 \\
3\end{array}$ & $\begin{array}{r}37.7 \\
9 \cdot 1 \\
25.0 \\
13.8 \\
38.5 \\
9.8 \\
19.0 \\
16.7\end{array}$ & $\begin{array}{l}3 \\
3 \\
5 \\
3 \\
6 \\
2 \\
4 \\
4\end{array}$ & $\begin{array}{r}14 \cdot 2 \\
13 \cdot 6 \\
25 \cdot 0 \\
13 \cdot 8 \\
28 \cdot 8 \\
9 \cdot 8 \\
19 \cdot 0 \\
22 \cdot 2\end{array}$ \\
\hline $\begin{array}{r}9 \\
10\end{array}$ & $\begin{array}{r}98 \\
1\end{array}$ & $\begin{array}{l}\mathrm{C}, \mathrm{T} \\
\mathrm{A}, \mathrm{T}, \mathrm{F}\end{array}$ & $\begin{array}{l}4 \cdot 3 \\
3 \cdot 0\end{array}$ & $\begin{array}{l}0.25 \\
0.20\end{array}$ & $\begin{array}{r}13 \\
9\end{array}$ & $\begin{array}{l}52.0 \\
46.2\end{array}$ & $\begin{array}{r}15 \\
7\end{array}$ & $\begin{array}{r}22 \\
9\end{array}$ & $\begin{array}{l}38 \\
18\end{array}$ & $\begin{array}{r}152.0 \\
92.3\end{array}$ & $\begin{array}{l}4 \\
3\end{array}$ & $\begin{array}{l}16.0 \\
15.4\end{array}$ & $\begin{array}{l}4 \\
4\end{array}$ & $\begin{array}{l}16.0 \\
20.5\end{array}$ \\
\hline $\begin{array}{l}\text { Mean } \\
\text { (1 SD) } \\
\text { Range }\end{array}$ & 13 & & $\begin{array}{l}3.25 \\
0.45\end{array}$ & $\begin{array}{l}0.21 \\
0.02\end{array}$ & $\begin{array}{r}10.2 \\
3.9\end{array}$ & $\begin{array}{l}49.0 \\
19.8\end{array}$ & & & $\begin{array}{l}19.5 \\
8.95\end{array}$ & $\begin{array}{l}91.94 \\
36.9\end{array}$ & $4 \cdot 2$ & $\begin{array}{l}20 \cdot 1 \\
10 \cdot 49\end{array}$ & 3.8 & $\begin{array}{r}18.29 \\
5.89\end{array}$ \\
\hline $\begin{array}{l}\text { Max } \\
\text { Min }\end{array}$ & $\begin{array}{r}98 \\
1\end{array}$ & & $\begin{array}{l}4 \cdot 3 \\
2 \cdot 7\end{array}$ & $\begin{array}{l}0.25 \\
0.18\end{array}$ & $\begin{array}{r}16 \\
2\end{array}$ & $\begin{array}{r}80.0 \\
9.8\end{array}$ & & & $\begin{array}{l}38 \\
10\end{array}$ & $\begin{array}{r}161.0 \\
47.2\end{array}$ & $\begin{array}{l}8 \\
2\end{array}$ & $\begin{array}{r}38.5 \\
9 \cdot 1\end{array}$ & $\begin{array}{l}6 \\
2\end{array}$ & $\begin{array}{r}28.8 \\
9.8\end{array}$ \\
\hline
\end{tabular}

A, acyanotic; Ao, aorta; Ao-S, aortic root at sinus level; Ao-ST, aortic root at sinotubular level; Ao-AA, ascending aorta; AP, anteroposterior; ASD, atrial septal defect: AV, aortic valve; BSA, body surface area; C, cyanosis; Coarctn, coarctation; CS, dilated coronary sinus; DA, ductus arteriosus; Desc, description; EFE, endocardial fibroelastosis; F, heart failure; IVS, interventricular septum; L, left; LAT, lateral; LV, left ventricle; LV contn, LV contraction; LVPW, posterior lef ventricular wall; M0, imperforate; M1, minimal mobility; M2, mobile; Musc VSD, muscular ventricular septal defect; MV, mitral valve annulus; RV, righ ventricle; RVH, right ventricular hypertrophy; SAX, short axis; SVC, superior vena cava; T, tachypnoea; TAPVD, total anomalous pulmonary venous drainage; Th, thickened.

Table 2 Clinical and echocardiographic features of the 15 neonates with other causes of right ventricular overload

\begin{tabular}{|c|c|c|c|c|c|c|c|c|c|c|c|c|c|}
\hline \multirow[b]{2}{*}{ Patient } & \multirow{2}{*}{$\begin{array}{l}\text { Age at } \\
\text { study } \\
\text { (days) }\end{array}$} & \multirow{2}{*}{$\begin{array}{l}\text { Weight } \\
\text { (kg) }\end{array}$} & \multirow{2}{*}{$\begin{array}{l}B S A \\
\left(m^{2}\right)\end{array}$} & \multicolumn{2}{|l|}{$L V$} & \multicolumn{2}{|l|}{$S A X$} & \multicolumn{2}{|l|}{$R V$} & \multicolumn{2}{|c|}{ LVPW } & \multicolumn{2}{|l|}{ IVS } \\
\hline & & & & $(m m)$ & $\left(m m / m^{2}\right)$ & $(A P)(\mathrm{mm})$ & $(L A T)(m m)$ & $(m m)$ & $\left(m m / m^{2}\right)$ & $(m m)$ & $\left(m m / m^{2}\right)$ & $(m m)$ & $\left(m m / m^{2}\right)$ \\
\hline $\begin{array}{l}1 \\
2\end{array}$ & $\begin{array}{r}2 \\
13\end{array}$ & $\begin{array}{l}2 \cdot 9 \\
3 \cdot 3\end{array}$ & $\begin{array}{l}0.21 \\
0.22\end{array}$ & $\begin{array}{l}17 \\
16\end{array}$ & $\begin{array}{l}82 \cdot 9 \\
74 \cdot 4\end{array}$ & $\begin{array}{l}10 \\
14\end{array}$ & $\begin{array}{l}13 \\
17\end{array}$ & $\begin{array}{l}15 \\
17\end{array}$ & $\begin{array}{l}73 \cdot 2 \\
79 \cdot 1\end{array}$ & $\begin{array}{l}4 \\
4\end{array}$ & $\begin{array}{l}19 \cdot 5 \\
18 \cdot 6\end{array}$ & $\begin{array}{l}4 \\
5\end{array}$ & $\begin{array}{l}19 \cdot 5 \\
23 \cdot 2\end{array}$ \\
\hline $\begin{array}{r}3 \\
4 \\
5 \\
6 \\
7 \\
8 \\
9 \\
10 \\
11 \\
12 \\
13 \\
14 \\
15\end{array}$ & $\begin{array}{r}1 \\
2 \\
10 \\
3 \\
3 \\
10 \\
3 \\
3 \\
2 \\
1 \\
1 \\
77 \\
8\end{array}$ & $\begin{array}{l}3 \cdot 0 \\
3 \cdot 5 \\
3 \cdot 1 \\
2 \cdot 6 \\
3 \cdot 6 \\
2 \cdot 7 \\
3 \cdot 5 \\
3 \cdot 3 \\
2 \cdot 8 \\
3 \cdot 0 \\
3 \cdot 5 \\
4 \cdot 1 \\
2 \cdot 6\end{array}$ & $\begin{array}{l}0.21 \\
0.22 \\
0.21 \\
0.19 \\
0.23 \\
0.20 \\
0.23 \\
0.21 \\
0.26 \\
0.22 \\
0.21 \\
0.25 \\
0.19\end{array}$ & $\begin{array}{r}17 \\
18 \\
21 \\
14 \\
18 \\
8 \\
14 \\
13 \\
21 \\
14 \\
11 \\
16 \\
13\end{array}$ & $\begin{array}{r}82.9 \\
80.7 \\
100.0 \\
72.9 \\
80.0 \\
40.0 \\
60.9 \\
61.9 \\
105.0 \\
63.6 \\
52.4 \\
64.0 \\
68.4\end{array}$ & $\begin{array}{r}14 \\
12 \\
15 \\
11 \\
16 \\
9 \\
13 \\
14 \\
14 \\
14 \\
13 \\
15 \\
11\end{array}$ & $\begin{array}{l}17 \\
14 \\
17 \\
15 \\
21 \\
14 \\
18 \\
18 \\
14 \\
20 \\
18 \\
17 \\
18\end{array}$ & $\begin{array}{l}15 \\
12 \\
15 \\
18 \\
17 \\
21 \\
21 \\
16 \\
18 \\
12 \\
15 \\
16 \\
13\end{array}$ & $\begin{array}{r}73.2 \\
53.8 \\
71.4 \\
93.7 \\
75.6 \\
105.0 \\
91.3 \\
76.2 \\
90.0 \\
54.5 \\
71.4 \\
64.0 \\
68.4\end{array}$ & $\begin{array}{l}4 \\
5 \\
5 \\
2 \\
4 \\
3 \\
3 \\
4 \\
4 \\
4 \\
4 \\
4 \\
3\end{array}$ & $\begin{array}{l}19.5 \\
22.4 \\
23.8 \\
10.4 \\
17.8 \\
15.0 \\
13.0 \\
19.0 \\
20.0 \\
18.2 \\
19.0 \\
16.0 \\
15.8\end{array}$ & $\begin{array}{l}6 \\
5 \\
6 \\
7 \\
5 \\
4 \\
4 \\
3 \\
6 \\
3 \\
4 \\
4 \\
3\end{array}$ & $\begin{array}{l}29.3 \\
22.4 \\
28.6 \\
36.5 \\
22.2 \\
20.0 \\
17.4 \\
14.3 \\
30.0 \\
13.6 \\
19.0 \\
16.0 \\
15.8\end{array}$ \\
\hline $\begin{array}{l}\text { Mean } \\
\text { (1 SD) } \\
\text { Range }\end{array}$ & 9 & $\begin{array}{l}3 \cdot 2 \\
0 \cdot 43\end{array}$ & $\begin{array}{l}0 \cdot 21 \\
0.02\end{array}$ & $\begin{array}{r}15.4 \\
3.5\end{array}$ & $\begin{array}{l}72.67 \\
16.97\end{array}$ & & & $\begin{array}{r}16 \cdot 1 \\
2 \cdot 7\end{array}$ & $\begin{array}{l}76 \cdot 06 \\
14 \cdot 16\end{array}$ & 3.8 & $\begin{array}{r}17 \cdot 88 \\
3 \cdot 42\end{array}$ & $4 \cdot 6$ & $\begin{array}{r}21.86 \\
6.63\end{array}$ \\
\hline $\begin{array}{l}\operatorname{Max} \\
\operatorname{Min}\end{array}$ & $\begin{array}{r}77 \\
1\end{array}$ & $\begin{array}{l}4 \cdot 1 \\
2 \cdot 6\end{array}$ & $\begin{array}{l}0.25 \\
0.19\end{array}$ & $\begin{array}{r}21 \\
8\end{array}$ & $\begin{array}{r}105 \cdot 0 \\
40.0\end{array}$ & & & $\begin{array}{l}21 \\
12\end{array}$ & $\begin{array}{r}105.0 \\
53.8\end{array}$ & $\begin{array}{l}5 \\
2\end{array}$ & $\begin{array}{l}23 \cdot 8 \\
10 \cdot 4\end{array}$ & $\begin{array}{l}7 \\
3\end{array}$ & $\begin{array}{l}36.5 \\
13.6\end{array}$ \\
\hline
\end{tabular}

dTGA, transposition of the great arteries; $N$, normal. Other abbreviations as in table 1 .

Table 3 Echocardiographic features of the 15 control neonates

\begin{tabular}{|c|c|c|c|c|c|c|c|c|c|c|c|c|c|}
\hline \multirow[b]{2}{*}{ Patient } & \multirow{2}{*}{$\begin{array}{l}\text { Age at } \\
\text { study } \\
\text { (days) }\end{array}$} & \multirow{2}{*}{$\begin{array}{l}\text { Weight } \\
\text { (kg) }\end{array}$} & \multirow{2}{*}{$\begin{array}{l}B S A \\
\left(m^{2}\right)\end{array}$} & \multicolumn{2}{|l|}{$L V$} & \multicolumn{2}{|l|}{$S A X$} & \multicolumn{2}{|l|}{$R V$} & \multicolumn{2}{|c|}{$L V P W$} & \multicolumn{2}{|l|}{ IVS } \\
\hline & & & & $(\mathrm{mm})$ & $\left(\mathrm{mm} / \mathrm{m}^{2}\right)$ & $(A P)(m m)$ & $(L A T)(\mathrm{mm})$ & $(\mathrm{mm})$ & $\left(\mathrm{mm} / \mathrm{m}^{2}\right)$ & $(\mathrm{mm})$ & $\left(m m / m^{2}\right)$ & $(m m)$ & $\left(\mathrm{mm} / \mathrm{m}^{2}\right)$ \\
\hline $\begin{array}{r}1 \\
2 \\
3 \\
4 \\
5 \\
6 \\
7 \\
8 \\
9 \\
10 \\
11 \\
12 \\
13 \\
14 \\
15\end{array}$ & $\begin{array}{r}14 \\
10 \\
11 \\
14 \\
5 \\
10 \\
60 \\
1 \\
7 \\
12 \\
2 \\
9 \\
15 \\
72 \\
38\end{array}$ & $\begin{array}{l}4.1 \\
3.8 \\
1.8 \\
3.9 \\
3.3 \\
3.0 \\
3.7 \\
2.0 \\
3.1 \\
3.1 \\
2.7 \\
3.4 \\
3.4 \\
3.3 \\
2.0\end{array}$ & $\begin{array}{l}0.24 \\
0.23 \\
0.17 \\
0.23 \\
0.22 \\
0.21 \\
0.23 \\
0.14 \\
0.20 \\
0.20 \\
0.20 \\
0.22 \\
0.22 \\
0.25 \\
0.14\end{array}$ & $\begin{array}{l}17 \\
15 \\
17 \\
17 \\
15 \\
16 \\
14 \\
12 \\
18 \\
15 \\
13 \\
15 \\
19 \\
12 \\
13\end{array}$ & $\begin{array}{r}72.3 \\
65.8 \\
101.2 \\
73.3 \\
69.8 \\
76.6 \\
60.9 \\
88.9 \\
92.3 \\
76.9 \\
65.0 \\
68.2 \\
86.4 \\
48.0 \\
96.3\end{array}$ & $\begin{array}{r}17 \\
17 \\
17 \\
18 \\
15 \\
15 \\
16 \\
8 \\
13 \\
14 \\
14 \\
14 \\
15 \\
18 \\
12\end{array}$ & $\begin{array}{r}17 \\
16 \\
17 \\
17 \\
15 \\
16 \\
18 \\
9 \\
18 \\
19 \\
15 \\
14 \\
15 \\
13 \\
12\end{array}$ & $\begin{array}{r}8 \\
8 \\
7 \\
11 \\
10 \\
7 \\
8 \\
7 \\
12 \\
12 \\
8 \\
14 \\
11 \\
15 \\
7\end{array}$ & $\begin{array}{l}34.0 \\
35.1 \\
41 \cdot 7 \\
47.4 \\
46.5 \\
33.5 \\
34.8 \\
51.9 \\
61.5 \\
61.5 \\
40.0 \\
63.6 \\
50.0 \\
60.0 \\
51.9\end{array}$ & $\begin{array}{l}4 \\
4 \\
4 \\
4 \\
3 \\
4 \\
3 \\
4 \\
3 \\
4 \\
4 \\
5 \\
3 \\
4 \\
3\end{array}$ & $\begin{array}{l}17 \cdot 0 \\
17 \cdot 5 \\
23 \cdot 8 \\
17 \cdot 2 \\
14 \cdot 0 \\
19 \cdot 1 \\
13 \cdot 0 \\
29 \cdot 6 \\
15 \cdot 4 \\
20 \cdot 5 \\
20 \cdot 0 \\
22 \cdot 7 \\
13 \cdot 6 \\
16 \cdot 0 \\
22 \cdot 2\end{array}$ & $\begin{array}{l}4 \\
4 \\
5 \\
5 \\
4 \\
4 \\
3 \\
4 \\
3 \\
3 \\
4 \\
5 \\
3 \\
3 \\
3\end{array}$ & $\begin{array}{l}17.0 \\
17.5 \\
29.8 \\
21.6 \\
18.6 \\
19.1 \\
13.0 \\
29.6 \\
15.4 \\
15.4 \\
20.0 \\
22.7 \\
13.6 \\
12.0 \\
22.2\end{array}$ \\
\hline $\begin{array}{l}\text { Mean } \\
\text { (1 SD) }\end{array}$ & 19 & $\begin{array}{l}3.1 \\
0.7\end{array}$ & $\begin{array}{l}0.20 \\
0.04\end{array}$ & $\begin{array}{r}15 \cdot 2 \\
2 \cdot 1\end{array}$ & $\begin{array}{l}76 \cdot 12 \\
14 \cdot 51\end{array}$ & & & $\begin{array}{l}9.7 \\
2.7\end{array}$ & $\begin{array}{l}47.56 \\
10 \cdot 82\end{array}$ & 3.7 & $\begin{array}{r}18 \cdot 79 \\
4.50\end{array}$ & $3 \cdot 8$ & $\begin{array}{r}19 \cdot 18 \\
5 \cdot 38\end{array}$ \\
\hline $\begin{array}{c}\text { Range } \\
\text { Max } \\
\text { Min }\end{array}$ & $\begin{array}{r}72 \\
1\end{array}$ & $\begin{array}{l}4 \cdot 1 \\
1.8\end{array}$ & $\begin{array}{l}0.25 \\
0.14\end{array}$ & $\begin{array}{l}19 \\
12\end{array}$ & $\begin{array}{r}101.2 \\
48.0\end{array}$ & & & $\begin{array}{r}15 \\
7\end{array}$ & $\begin{array}{l}63.6 \\
33.5\end{array}$ & $\begin{array}{l}5 \\
3\end{array}$ & $\begin{array}{l}29.6 \\
13.0\end{array}$ & $\begin{array}{l}5 \\
3\end{array}$ & $\begin{array}{l}29.8 \\
12.0\end{array}$ \\
\hline
\end{tabular}




\begin{tabular}{|c|c|c|c|c|c|c|c|c|c|c|c|}
\hline \multicolumn{2}{|l|}{$M V$} & \multirow{2}{*}{$\begin{array}{l}M V \\
\operatorname{desc}\end{array}$} & \multicolumn{2}{|l|}{$A o-S$} & \multicolumn{2}{|c|}{$A o-S T$} & \multicolumn{2}{|c|}{$A o-A A$} & \multirow{2}{*}{$\begin{array}{l}A V \\
\text { desc }\end{array}$} & \multirow{2}{*}{$\begin{array}{l}L V \text { contn } \\
(1-3)\end{array}$} & \multirow[b]{2}{*}{ Other features } \\
\hline$(\mathrm{mm})$ & $\left(\mathrm{mm} / \mathrm{m}^{2}\right)$ & & $(\mathrm{mm})$ & $\left(\mathrm{mm} / \mathrm{m}^{2}\right)$ & $(m m)$ & $\left(\mathrm{mm} / \mathrm{m}^{2}\right)$ & $(\mathrm{mm})$ & $\left(\mathrm{mm} / \mathrm{m}^{2}\right)$ & & & \\
\hline $\begin{array}{l}4 \\
5 \\
8 \\
4 \\
0 \\
0 \\
4 \\
6\end{array}$ & $\begin{array}{c}18 \cdot 9 \\
22 \cdot 7 \\
40 \cdot 0 \\
18 \cdot 3 \\
0 \\
0 \\
19 \cdot 0 \\
33 \cdot 3\end{array}$ & $\begin{array}{l}\text { Th, M1 } \\
\text { M1 } \\
\text { M2 } \\
\text { M1 } \\
\text { M0 } \\
\text { M0 } \\
\text { Th, M1 } \\
\text { M2 }\end{array}$ & $\begin{array}{l}3 \\
3 \\
5 \\
3 \\
5 \\
4 \\
2 \\
6\end{array}$ & $\begin{array}{r}14 \cdot 2 \\
13.6 \\
25.0 \\
13.8 \\
24.0 \\
19.5 \\
9.5 \\
33.3\end{array}$ & $\begin{array}{l}3 \\
3 \\
6 \\
3 \\
5 \\
4 \\
2 \\
4\end{array}$ & $\begin{array}{r}14 \cdot 2 \\
13.6 \\
30 \cdot 0 \\
13 \cdot 8 \\
24 \cdot 0 \\
19.5 \\
9.5 \\
22 \cdot 2\end{array}$ & $\begin{array}{l}3 \\
3 \\
9 \\
5 \\
5 \\
5 \\
3 \\
5\end{array}$ & $\begin{array}{l}14 \cdot 2 \\
13 \cdot 6 \\
45 \cdot 0 \\
22 \cdot 9 \\
24 \cdot 0 \\
24 \cdot 4 \\
14 \cdot 3 \\
27 \cdot 8\end{array}$ & $\begin{array}{l}\text { Th, M1 } \\
\text { M2 } \\
\text { M2 } \\
\text { Th, M1 } \\
\text { M2 } \\
\text { M0 } \\
\text { M0 } \\
\text { M2 }\end{array}$ & $\begin{array}{l}1 \\
1 \\
2 \\
1 \\
1 \\
1 \\
1 \\
2\end{array}$ & $\begin{array}{l}\text { Th posterior wall, musc VSD, RVH, DA } \\
\text { RVH } \\
\text { Musc VSD, RVH } \\
\text { RVH } \\
\text { Th posterior wall, bicuspid AV, RVH } \\
\text { LV slit like cavity, RVH, ASD, DA } \\
\text { RVH, DA } \\
\text { DA, ASD, RVH, very dilated main PA, } \\
\text { L SVC to CS }\end{array}$ \\
\hline $\begin{array}{r}10 \\
5\end{array}$ & $\begin{array}{l}40 \cdot 0 \\
25 \cdot 6\end{array}$ & $\begin{array}{l}\text { M2 } \\
\text { M2 }\end{array}$ & $\begin{array}{l}8 \\
3\end{array}$ & $\begin{array}{l}32 \cdot 0 \\
15 \cdot 4\end{array}$ & $\begin{array}{l}7 \\
8\end{array}$ & $\begin{array}{l}28 \cdot 0 \\
41 \cdot 0\end{array}$ & $\begin{array}{l}8 \\
8\end{array}$ & $\begin{array}{l}32 \cdot 0 \\
41 \cdot 0\end{array}$ & $\begin{array}{l}\mathrm{M} 2 \\
\mathrm{Th}, \mathrm{M1}\end{array}$ & $\begin{array}{l}3 \\
2\end{array}$ & $\begin{array}{l}\text { TAPVD, RV:EFE } \\
\text { Ao coarctn + DA repaired }\end{array}$ \\
\hline $\begin{array}{l}5 \cdot 8 \\
2 \cdot 2\end{array}$ & $\begin{array}{r}27 \cdot 3 \\
9 \cdot 3\end{array}$ & & $\begin{array}{l}4 \cdot 2 \\
1 \cdot 8\end{array}$ & $\begin{array}{r}20 \cdot 03 \\
8 \cdot 21\end{array}$ & $\begin{array}{l}4.5 \\
1.96\end{array}$ & $\begin{array}{r}21 \cdot 58 \\
9.57\end{array}$ & $\begin{array}{l}5 \cdot 4 \\
2 \cdot 22\end{array}$ & $\begin{array}{l}25.92 \\
10.91\end{array}$ & & & \\
\hline $\begin{array}{r}10 \\
0\end{array}$ & $\begin{array}{l}40 \cdot 0 \\
18 \cdot 3\end{array}$ & & $\begin{array}{l}8 \\
2\end{array}$ & $\begin{array}{r}33 \cdot 3 \\
9 \cdot 5\end{array}$ & $\begin{array}{l}8 \\
2\end{array}$ & $\begin{array}{r}41 \cdot 0 \\
9 \cdot 5\end{array}$ & $\begin{array}{l}9 \\
3\end{array}$ & $\begin{array}{l}45 \cdot 0 \\
13 \cdot 6\end{array}$ & & & \\
\hline
\end{tabular}

\begin{tabular}{|c|c|c|c|c|c|c|c|c|c|c|c|}
\hline \multicolumn{2}{|l|}{$M V$} & \multirow{2}{*}{$\begin{array}{l}M V \\
\text { desc }\end{array}$} & \multicolumn{2}{|l|}{$A o-S$} & \multicolumn{2}{|c|}{$A o-S T$} & \multicolumn{2}{|c|}{$A o-A A$} & \multirow{2}{*}{$\begin{array}{l}A V \\
\operatorname{desc}\end{array}$} & \multirow{2}{*}{$\begin{array}{l}L V \text { contn } \\
(1-3)\end{array}$} & \multirow[b]{2}{*}{ Diagnosis } \\
\hline$(m m)$ & $\left(\mathrm{mm} / \mathrm{m}^{2}\right)$ & & $(m m)$ & $\left(\mathrm{mm} / \mathrm{m}^{2}\right)$ & $(\mathrm{mm})$ & $\left(m m / m^{2}\right)$ & $(\mathrm{mm})$ & $\left(\mathrm{mm} / \mathrm{m}^{2}\right)$ & & & \\
\hline $\begin{array}{r}9 \\
10\end{array}$ & $\begin{array}{l}43.9 \\
46.5\end{array}$ & $\begin{array}{l}\mathbf{N} \\
\mathbf{N}\end{array}$ & $\begin{array}{l}7 \\
6\end{array}$ & $\begin{array}{l}34 \cdot 1 \\
27 \cdot 9\end{array}$ & $\begin{array}{l}7 \\
6\end{array}$ & $\begin{array}{l}34 \cdot 1 \\
27 \cdot 9\end{array}$ & $\begin{array}{l}7 \\
6\end{array}$ & $\begin{array}{l}34 \cdot 1 \\
27 \cdot 9\end{array}$ & $\begin{array}{l}\mathbf{N} \\
\mathbf{N}\end{array}$ & $\begin{array}{l}3 \\
3\end{array}$ & $\begin{array}{l}\text { TAPVD, CS } \\
\text { VSD (subarterial), subaortic stenosis, Ao } \\
\text { coarctn, CS }\end{array}$ \\
\hline $\begin{array}{r}10 \\
10 \\
12 \\
10 \\
12 \\
8 \\
9 \\
11 \\
9 \\
9 \\
11 \\
13 \\
8\end{array}$ & $\begin{array}{l}48 \cdot 8 \\
44 \cdot 8 \\
57 \cdot 1 \\
52 \cdot 1 \\
53 \cdot 3 \\
40 \cdot 0 \\
39 \cdot 1 \\
52 \cdot 4 \\
45 \cdot 0 \\
40 \cdot 9 \\
52 \cdot 4 \\
52 \cdot 0 \\
42 \cdot 1\end{array}$ & $\begin{array}{l}\mathbf{N} \\
\mathbf{N} \\
\mathbf{N} \\
\mathbf{N} \\
\mathbf{N} \\
\mathbf{N} \\
\mathbf{N} \\
\mathbf{N} \\
\mathbf{N} \\
\mathbf{N} \\
\mathbf{N} \\
\mathbf{N} \\
\mathbf{N}\end{array}$ & $\begin{array}{r}10 \\
10 \\
8 \\
8 \\
9 \\
7 \\
6 \\
7 \\
10 \\
8 \\
9 \\
11 \\
9\end{array}$ & $\begin{array}{l}48 \cdot 8 \\
44 \cdot 8 \\
38 \cdot 1 \\
41 \cdot 7 \\
40 \cdot 0 \\
35 \cdot 0 \\
26 \cdot 1 \\
33 \cdot 3 \\
50 \cdot 0 \\
36 \cdot 4 \\
42 \cdot 9 \\
44 \cdot 0 \\
47 \cdot 4\end{array}$ & $\begin{array}{r}11 \\
10 \\
9 \\
7 \\
9 \\
6 \\
6 \\
6 \\
10 \\
7 \\
8 \\
10 \\
9\end{array}$ & $\begin{array}{l}53.7 \\
44.8 \\
42.9 \\
36.5 \\
40.0 \\
30.0 \\
26.1 \\
28.6 \\
50.0 \\
31.8 \\
38.1 \\
40.0 \\
47.4\end{array}$ & $\begin{array}{r}11 \\
10 \\
11 \\
8 \\
11 \\
7 \\
7 \\
6 \\
10 \\
8 \\
10 \\
11 \\
8\end{array}$ & $\begin{array}{l}53 \cdot 7 \\
44.8 \\
52.4 \\
41.7 \\
48.9 \\
35 \cdot 0 \\
30 \cdot 4 \\
28 \cdot 6 \\
50.0 \\
36.4 \\
47.6 \\
44.0 \\
42.1\end{array}$ & $\begin{array}{l}\mathbf{N} \\
\mathbf{N} \\
\mathbf{N} \\
\mathbf{N} \\
\mathbf{N} \\
\mathbf{N} \\
\mathbf{N} \\
\mathbf{N} \\
\mathbf{N} \\
\mathbf{N} \\
\mathbf{N} \\
\mathbf{N} \\
\mathbf{N}\end{array}$ & $\begin{array}{l}3 \\
3 \\
3 \\
3 \\
3 \\
3 \\
3 \\
3 \\
3 \\
3 \\
3 \\
3 \\
3\end{array}$ & $\begin{array}{l}\text { dTGA, RVH, CS } \\
\text { Ebstein, musc VSD } \\
\text { Ao coarctn, musc VSD } \\
\text { Persistent fetal circulation } \\
\text { TAPVD } \\
\text { TAPVD, impaired RV function } \\
\text { ASD } \\
\text { Ao coarctn } \\
\text { dTGA } \\
\text { TAPVD } \\
\text { VSD, ASD } \\
\text { dTGA (VSD + ASD) } \\
\text { ASD }\end{array}$ \\
\hline $\begin{array}{r}10 \cdot 1 \\
1.5\end{array}$ & $\begin{array}{r}47 \cdot 37 \\
5 \cdot 63\end{array}$ & & $\begin{array}{l}8 \cdot 3 \\
1 \cdot 5\end{array}$ & $\begin{array}{r}39 \cdot 36 \\
7 \cdot 25\end{array}$ & $\begin{array}{l}8 \cdot 1 \\
1 \cdot 8\end{array}$ & $\begin{array}{r}38 \cdot 12 \\
8 \cdot 48\end{array}$ & $\begin{array}{l}8.7 \\
1.9\end{array}$ & $\begin{array}{r}41 \cdot 17 \\
8 \cdot 63\end{array}$ & & & \\
\hline $\begin{array}{r}13 \\
8\end{array}$ & $\begin{array}{l}57 \cdot 1 \\
39 \cdot 1\end{array}$ & & $\begin{array}{r}11 \\
6\end{array}$ & $\begin{array}{l}50 \cdot 0 \\
26 \cdot 1\end{array}$ & $\begin{array}{r}11 \\
6\end{array}$ & $\begin{array}{l}53 \cdot 7 \\
26.1\end{array}$ & $\begin{array}{r}11 \\
6\end{array}$ & $\begin{array}{l}53.7 \\
27.9\end{array}$ & & & \\
\hline
\end{tabular}

\begin{tabular}{|c|c|c|c|c|c|c|c|}
\hline \multicolumn{2}{|l|}{$M V$} & \multicolumn{2}{|l|}{$A o-S$} & \multicolumn{2}{|l|}{$A o-S T$} & \multicolumn{2}{|c|}{$A 0-A A$} \\
\hline$(\mathrm{mm})$ & $\left(\mathrm{mm} / \mathrm{m}^{2}\right)$ & $(m m)$ & $\left(\mathrm{mm} / \mathrm{m}^{2}\right)$ & $(m m)$ & $\left(\mathrm{mm} / \mathrm{m}^{2}\right)$ & $(m m)$ & $\left(m m / m^{2}\right)$ \\
\hline $\begin{array}{r}10 \\
8 \\
11 \\
12 \\
9 \\
11 \\
10 \\
12 \\
12 \\
11 \\
8 \\
10 \\
10 \\
10 \\
7\end{array}$ & $\begin{array}{l}42.6 \\
35 \cdot 1 \\
65.5 \\
51 \cdot 7 \\
41.9 \\
52 \cdot 6 \\
43.5 \\
88.9 \\
61.5 \\
56 \cdot 4 \\
40.0 \\
45.5 \\
45.5 \\
40.0 \\
51.9\end{array}$ & $\begin{array}{l}9 \\
7 \\
8 \\
9 \\
9 \\
8 \\
8 \\
6 \\
6 \\
7 \\
6 \\
9 \\
9 \\
8 \\
7\end{array}$ & $\begin{array}{l}38 \cdot 3 \\
30 \cdot 7 \\
47 \cdot 6 \\
38 \cdot 8 \\
41.9 \\
38 \cdot 3 \\
34 \cdot 8 \\
44 \cdot 4 \\
30 \cdot 8 \\
35.9 \\
30 \cdot 0 \\
40 \cdot 9 \\
40 \cdot 9 \\
32 \cdot 0 \\
51.9\end{array}$ & $\begin{array}{l}7 \\
8 \\
7 \\
8 \\
8 \\
8 \\
8 \\
7 \\
5 \\
6 \\
8 \\
9 \\
9 \\
7 \\
7\end{array}$ & $\begin{array}{l}29.8 \\
35.1 \\
41.7 \\
34.5 \\
37.2 \\
38.3 \\
34.8 \\
51.9 \\
25.6 \\
30.8 \\
40.0 \\
40.9 \\
40.9 \\
28.0 \\
51.9\end{array}$ & $\begin{array}{r}9 \\
8 \\
7 \\
10 \\
10 \\
9 \\
8 \\
8 \\
7 \\
6 \\
7 \\
10 \\
10 \\
9 \\
8\end{array}$ & $\begin{array}{l}38.3 \\
35.1 \\
41.7 \\
43.1 \\
46.5 \\
43.1 \\
34.8 \\
59.3 \\
35.9 \\
30.8 \\
35.0 \\
45.5 \\
45.5 \\
36.0 \\
59.3\end{array}$ \\
\hline $\begin{array}{r}10.1 \\
1.5\end{array}$ & $\begin{array}{l}50 \cdot 83 \\
13.51\end{array}$ & $\begin{array}{l}7 \cdot 7 \\
1 \cdot 16\end{array}$ & $\begin{array}{r}38.47 \\
6.41\end{array}$ & $\begin{array}{l}7.5 \\
1.1\end{array}$ & $\begin{array}{r}37.42 \\
7.64\end{array}$ & $\begin{array}{l}8 \cdot 3 \\
1 \cdot 3\end{array}$ & $\begin{array}{r}41.97 \\
8.43\end{array}$ \\
\hline $\begin{array}{r}12 \\
7\end{array}$ & $\begin{array}{l}88.9 \\
35 \cdot 1\end{array}$ & $\begin{array}{l}9 \\
6\end{array}$ & $\begin{array}{l}51.9 \\
30.0\end{array}$ & $\begin{array}{l}9 \\
5\end{array}$ & $\begin{array}{l}51.9 \\
25.6\end{array}$ & $\begin{array}{r}10 \\
6\end{array}$ & $\begin{array}{l}59 \cdot 3 \\
30.8\end{array}$ \\
\hline
\end{tabular}

measured at end systole. For each, three measurements were taken from three successive cardiac cycles. The mean value was expressed as an absolute value $(\mathrm{mm})$ and corrected for body surface area $\left(\mathrm{mm} / \mathrm{m}^{2}\right)$.

To assess the degree of geometric distortion of the ventricular cavity, we measured the percentage difference between the anteroposterior and lateral dimensions of each left ventricular cavity in the short axis projection. These percentages were used an an index of distortion; the value for a perfect circle is $0 \%$.

Left ventricular systolic function was assessed from the real time image by two observers experienced in neonatal studies. Each blindly reviewed all the studies from the three groups. Systolic contraction, evaluated from the serial short axis left ventricular 
(A) Parasternal long axis

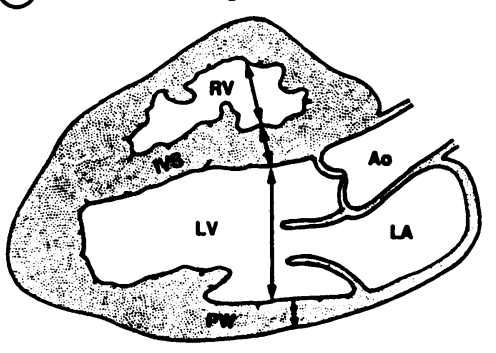

(B) Parasternal short axis

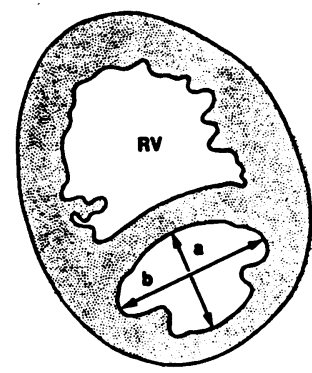

(C) High left parasternal

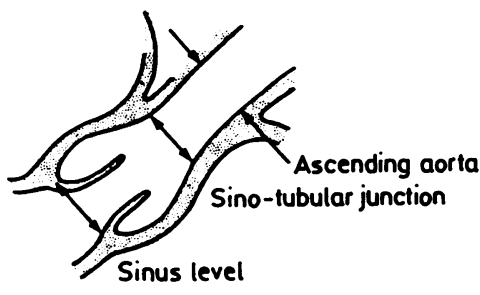

(D) Apical four-chamber Mitral valve annulus

Figure 1 Diagrammatic representation of the measurements made. (A) Parasternal long axis projection at end diastole. (B) Cross sectional short axis projection at end diastole. (C) Parasternal long axis projection of the aortic root at end systole. (D) Apical four chamber projection.

projections, was graded from 1 to 3 ( $1=$ negligible contractile function; $2=$ reduced but fair systolic contractile motion; and $3=$ normal contractile function). Disagreement between observers was settled reaching a consensus after a joint review of the study in question.

Tables 1 and 2 show the associated congenial heart defects.

\section{STATISTICAL ANALYSIS}

All results are corrected for body surface area, and dimensions are quoted as mean (1 SD). We compared the samples using Student's ' $t$ distribution. A probability value of $<0.05$ was regarded as significant.

We used logistic regression to produce a classification rule based on the dimension of the left ventricular cavity, mitral valve annulus, and aortic root to estimate the probability of a neonate having hypoplastic left heart syndrome. Such a rule can be formed from any set of the measurements taken and the more informative a set of measurements is the better will be the classification rule obtained from it.

To assess the performance of a classification rule one could simply count the number of neonates correctly classified, but this does not measure the uncertainty with which each individual has been classified. Such information is provided by a statistic known as the deviance. ${ }^{14}$ When a number of measurements are added to the rule, the greater the decrease in the deviance, the greater the improvement in the rule. Significance was assessed by refering the decrease in deviance to the $\chi^{2}$ distribution with degrees of freedom equal to the number of measurements added. The analysis was carried out using the statistical package Generalised Linear Interactive Modelling. ${ }^{15}$

\section{Results}

In all individuals (patients and controls) echocardiograms were good enough to show the complete intracardiac anatomy. Tables 1 and 2 summarise the clinical features of the 10 neonates with hypoplastic left heart syndrome and the 15 neonates with other causes of right ventricular overload. Cardiac failure was the major presenting symptom in both groups; six $(60 \%)$ of the neonates with hypoplastic left heart syndrome were acyanotic.
CROSS SECTIONAL ECHOCARDIOGRAPHIC FEATURES

Left ventricular cavity (fig 2)

Although patients with hypoplastic left heart syndrome had smaller left ventricular cavities than patients with other causes of right ventricular overload $(p<0.005)$ and the normal individuals $(p<0.001)$ as a whole, there was a considerable overlap of individual values among the three groups.

There was no significant difference in the degree of distortion between the patients with hypoplastic left heart syndrome and those with other causes of right ventricular overload $(25 \cdot 4 \%, 95 \%$ confidence interval $12 \cdot 1$ to $38 \cdot 7)$ compared with $29.9 \%(20 \cdot 6$ to $39 \cdot 2)$. The normal controls showed much less distortion $(10.9 \%, 2.9-18.9)$.

In all but one neonate with hypoplastic left heart syndrome left ventricular contractile function was poorer than in the subjects with right ventricular overload, all of whom had normal function (tables 1 and 2).

Right ventricular cavity size (fig 2)

In patients with hypoplastic left heart syndrome the size of the right ventricular cavity was similar to that in patients with other causes of right ventricular overload. In both groups, however, the diameter was greater than that in the controls $(\mathrm{p}<0.001)$.

\section{Mitral valve annulus (fig 2)}

The mitral valve annulus was smaller in patients with hypoplastic left heart syndrome than in patients with other causes of right ventricular overload $(p<0.001)$ and in the controls $(p<0.001)$. Only two neonates with hypoplastic left heart syndrome had a mitral valve annulus within the range of values for the group with right ventricular overload and controls, while in two other patients with hypoplastic left heart syndrome the mitral valve was clearly imperforate.

\section{Aorta (fig 3)}

The dimensions of the aortic root and ascending aorta were smaller in patients with hypoplastic left heart syndrome than in those with right ventricular overload ( $p<0.001)$, though there was a considerable spread of values. This spread was also seen in the control group. 
Appearance of the aortic and mitral valves Seven $(70 \%)$ of the neonates with hypoplastic left heart syndrome had abnormalities of the aortic and mitral valves ranging from imperforate valves (two of 10 ) to thickened valves with reduced mobility (four of 10); in three patients both the left heart valves were of normal appearance. One of these three had coexistent supracardiac total anomalous pulmonary venous drainage with obstruction (patient 9). These findings were confirmed at necropsy.

No patient with a right ventricular loading condition had a morphologically abnormal mitral or aortic valve.

Wall thickness (tables 1-3)

Left ventricular wall thickness was generally similar in the three groups of patients. Two patients ( 1 and 5) with hypoplastic left heart syndrome showed considerable thickening of
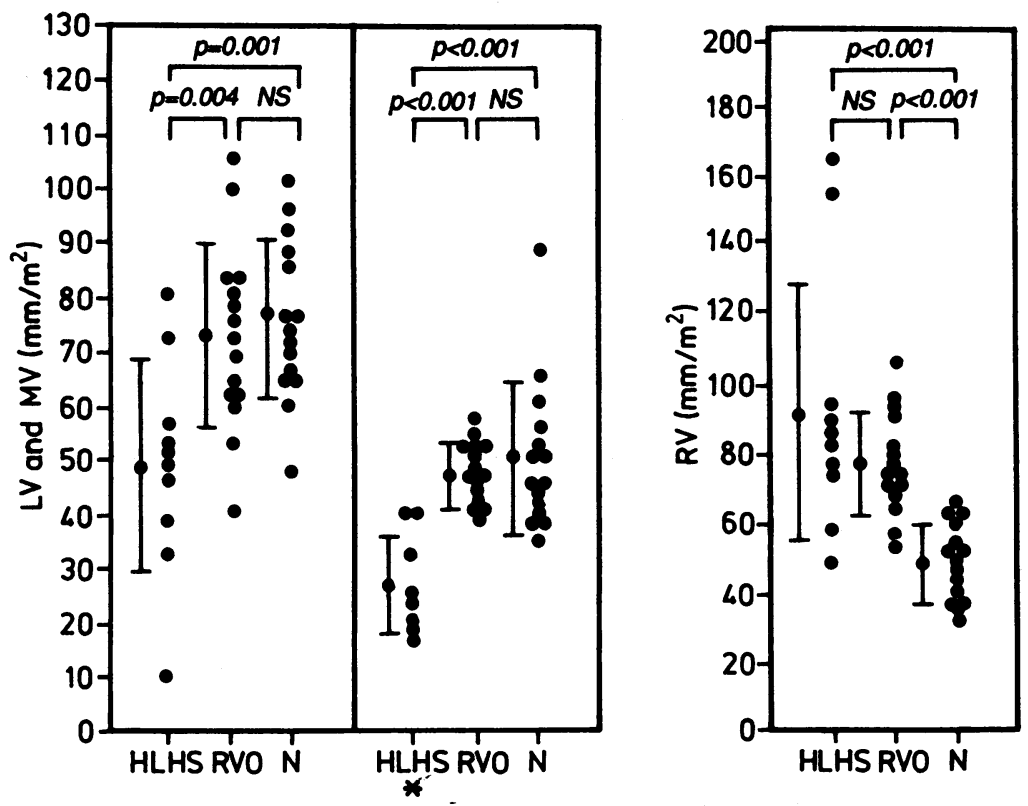

Figure 2 Comparative values and mean (1SD) for the neonates with hypoplastic left heart syndrome ( $H L H S)$, right ventricular overload (RVO), and normal neonates $(N)$ at left ventricular $(L V)$, mitral annulus $(M V)$, and right ventricular $(R V)$ levels. ${ }^{\star}$ Two HLHS patients had imperforate mitral valves.

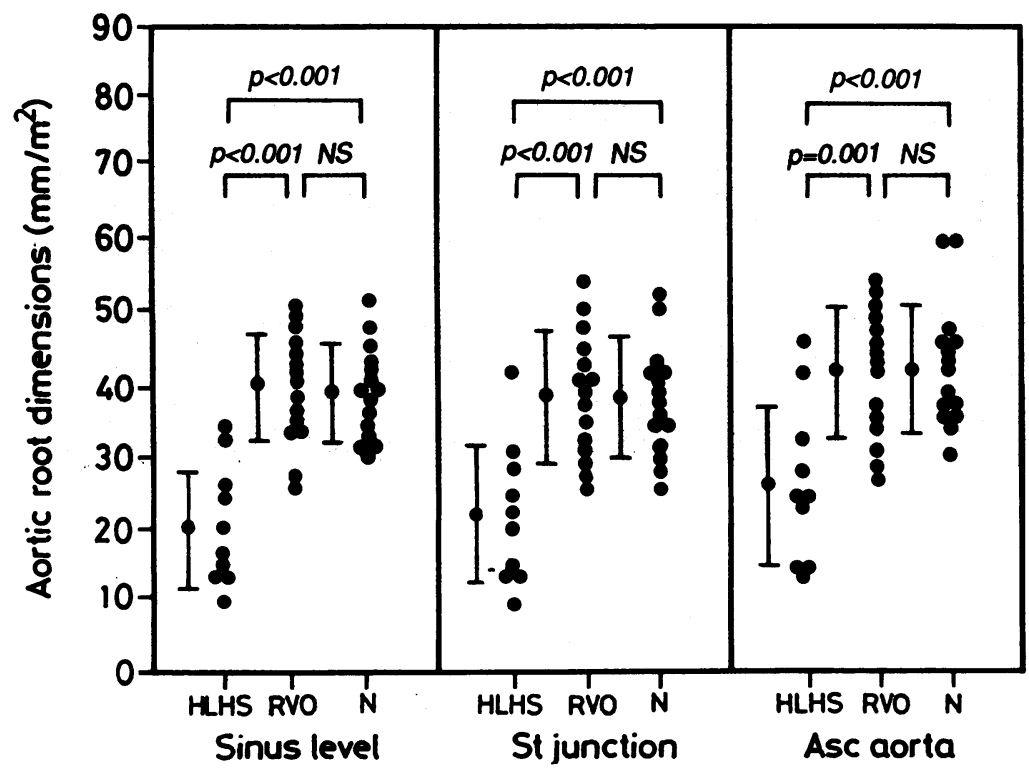

Figure 3 Comparative values and means (1SD) for aortic root dimensions at the sinus level, the sinotubular junction ( $S t$ ), and in the ascending aorta (Asc aorta).

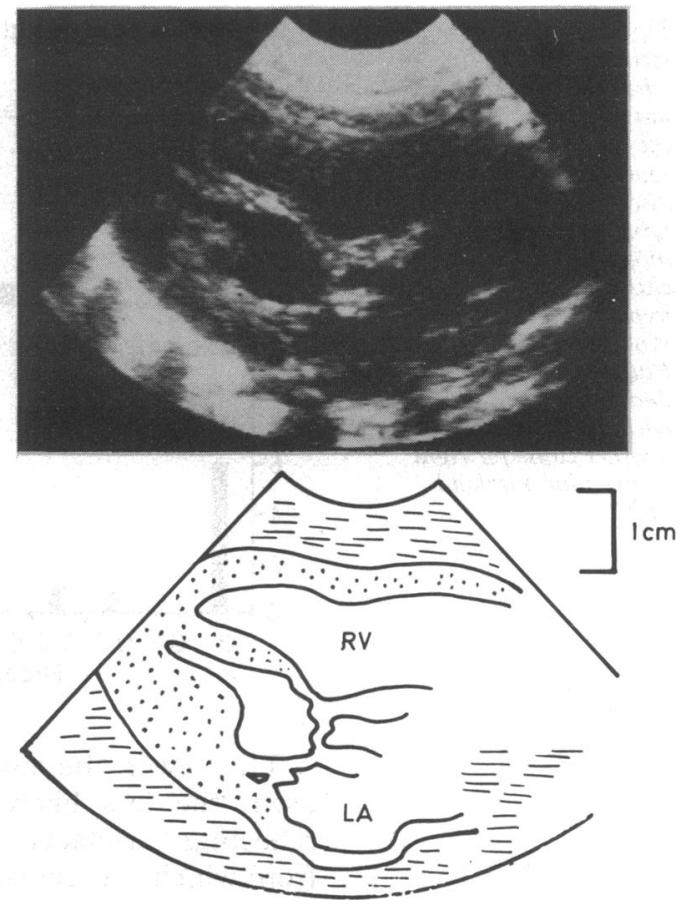

Figure 4 Parasternal long axis view in patient 5 with hypoplastic left heart syndrome showing the thickened appearance of the posterior wall.

the posterior wall (fig 4). This feature was noted at necropsy and described as fibroelastic thickening.

\section{Associated lesions (table 1)}

In four of the patients with hypoplastic left heart syndrome there were additional malformations that could have produced right ventricular loading. Patient 9 had total anomalous pulmonary venous drainage with obstruction, two others (patients 6 and 8) had secundum atrial septal defects, and one (patient 10) had an aortic coarctation.

\section{Statistical analysis}

Logistic regression showed that the diameter of the mitral valve annulus was the single best measurement for distinguishing patients with hypoplastic left heart syndrome from either healthy controls or those with other causes of right ventricular overload. It correctly identified 37 of the 40 neonates (fig 5). The diameter of the aortic root at sinus level was the only other single measurement that correctly classified as many as this, but the deviance was greater (15.79 compared with 12.33), indicating a less good fit to the data-as was evident from the histogram of estimated probabilities (fig 5).

Of all the rules that used two measurements, none misclassified less than three individuals, although the rule using mitral valve annulus, aortic root measurement at the sino-tubular junction, and the dimension of the ascending aorta misclassified only one. However, the decrease in deviance from the model using only mitral valve annulus diameter was $5 \cdot 15 \%$, which when referred to a $\chi^{2}$ distribution with 2 degrees of freedom is not significant at the 5\% level. This indicates that such an improvement in the classification rule could well have arisen by chance. No rule classified all 40 neonates correctly. 

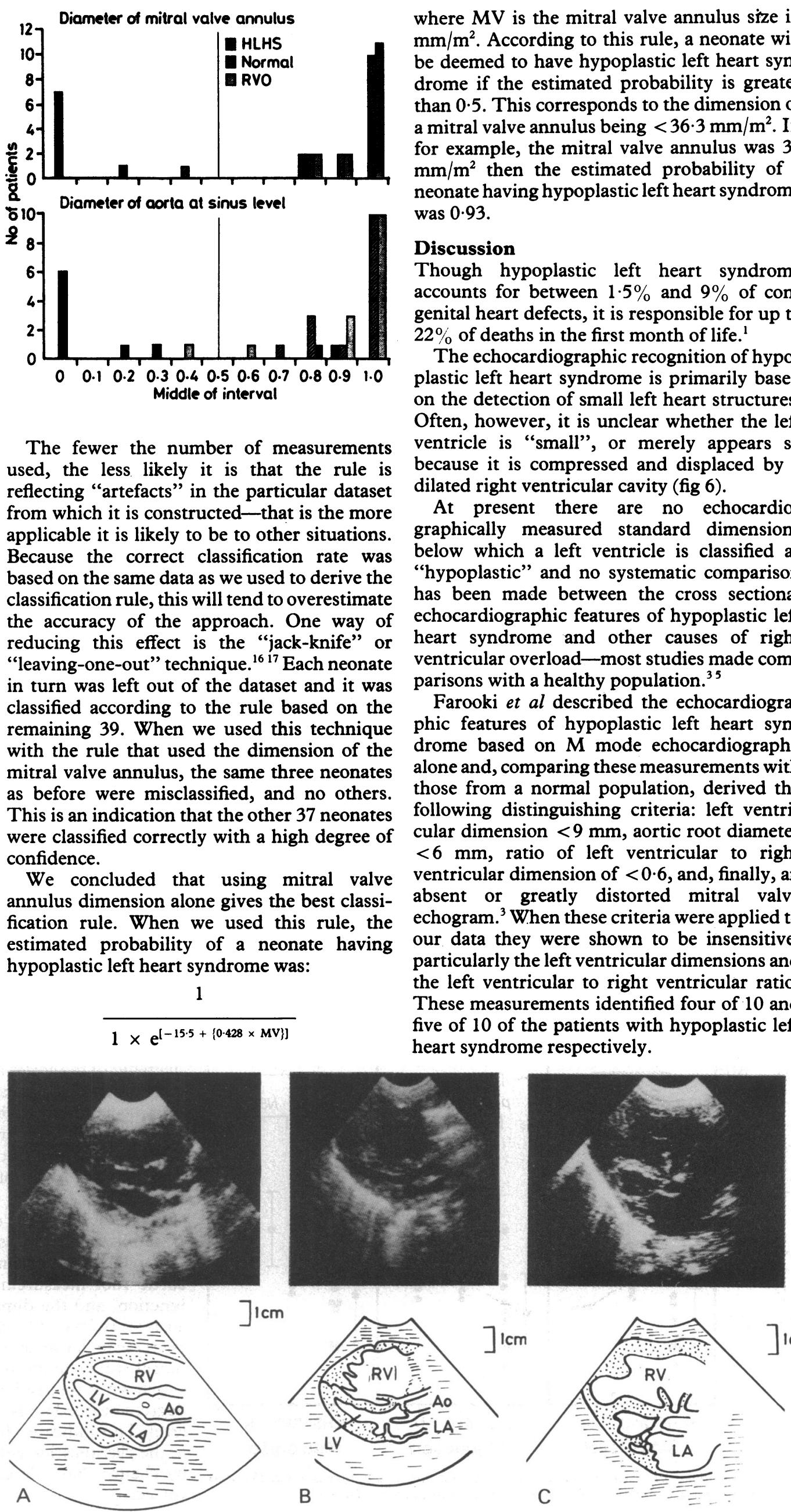

B

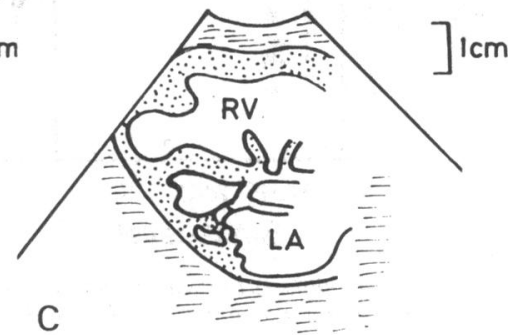
$\mathrm{mm} / \mathrm{m}^{2}$ then the estimated probability of a
Figure 5 Histograms of estimated probabilities when the diameter of the mitral annulus (top) and the diameter of the aortic root (bottom) were used as discriminants. Patients lying between probability intervals 0 and 0.5 are classified as having hypoplastic left heart syndrome (HLHS). Patients lying between 0.5 and 1.0 are classified as normal or as having another cause for right ventricular overload (RVO).
The fewer the number of measurements used, the less likely it is that the rule is reflecting "artefacts" in the particular dataset from which it is constructed-that is the more applicable it is likely to be to other situations. Because the correct classification rate was based on the same data as we used to derive the classification rule, this will tend to overestimate the accuracy of the approach. One way of reducing this effect is the "jack-knife" or "leaving-one-out" technique. ${ }^{16}{ }^{17}$ Each neonate in turn was left out of the dataset and it was classified according to the rule based on the remaining 39 . When we used this technique with the rule that used the dimension of the mitral valve annulus, the same three neonates as before were misclassified, and no others. This is an indication that the other 37 neonates were classified correctly with a high degree of confidence.

We concluded that using mitral valve annulus dimension alone gives the best classification rule. When we used this rule, the estimated probability of a neonate having hypoplastic left heart syndrome was:

1

$1 \times \mathrm{e}^{[-15.5+\{0.428 \times M V\}]}$

where $\mathrm{MV}$ is the mitral valve annulus size in $\mathrm{mm} / \mathrm{m}^{2}$. According to this rule, a neonate will be deemed to have hypoplastic left heart syndrome if the estimated probability is greater than $0 \cdot 5$. This corresponds to the dimension of a mitral valve annulus being $<36.3 \mathrm{~mm} / \mathrm{m}^{2}$. If, for example, the mitral valve annulus was 30 neonate having hypoplastic left heart syndrome was 0.93 .

\section{Discussion}

hypoplastic left heart syndrc accounts for between $1.5 \%$ and $9 \%$ of congenital heart defects, it is responsible for up to $22 \%$ of deaths in the first month of life. ${ }^{1}$

The echocardiographic recognition of hypoplastic left heart syndrome is primarily based on the detection of small left heart structures. Often, however, it is unclear whether the left ventricle is "small", or merely appears so because it is compressed and displaced by a dilated right ventricular cavity (fig 6).

At present there are no echocardiographically measured standard dimensions below which a left ventricle is classified as "hypoplastic" and no systematic comparison has been made between the cross sectional echocardiographic features of hypoplastic left heart syndrome and other causes of right ventricular overload-most studies made comparisons with a healthy population. ${ }^{35}$

Farooki et al described the echocardiographic features of hypoplastic left heart syndrome based on $M$ mode echocardiography alone and, comparing these measurements with those from a normal population, derived the following distinguishing criteria: left ventricular dimension $<9 \mathrm{~mm}$, aortic root diameter $<6 \mathrm{~mm}$, ratio of left ventricular to right ventricular dimension of $<0.6$, and, finally, an absent or greatly distorted mitral valve echogram. ${ }^{3}$ When these criteria were applied to our data they were shown to be insensitive, particularly the left ventricular dimensions and the left ventricular to right ventricular ratio. These measurements identified four of 10 and five of 10 of the patients with hypoplastic left heart syndrome respectively.

Figure 6 Parasternal long axis views comparin with a neonate with right ventricular overload (B) and a neonate with syndrome $(C)$. 
The limitations of $M$ mode echocardiographic measurements in the diagnosis of hypoplastic left heart syndrome have also been highlighted by Lange et al who assessed $M$ mode echocardiographic, cross sectional echocardiographic, and necropsy correlations. $^{18}$ They found that the range of hypoplastic left heart syndrome was too great to be resolved by $M$ mode echocardiographic measurements alone, but the correlation between cross sectional echocardiography and postmortem findings was close.

In our study the variability in left ventricular cavity dimension in hypoplastic left heart syndrome measured from cross sectional studies was too great to distinguish between this condition and other causes of right ventricular overload or between the normal controls. Only three of the 10 neonates with hypoplastic left heart had left ventricular cavity sizes outside the range of the group with right ventricular loading.

The dimension of the aortic root at sinus level was a more discriminative variable; however, coexistent aortic coarctation or an interrupted aortic arch will cause diagnostic confusion as reported by Lange et al. ${ }^{18}$ This condition did not occur in our population. The diameter of the mitral valve annulus was the most discriminative single variable and correctly classified all but three of the 40 neonates.

In our series, the three patients with morphologically normal left heart valves posed the most difficult diagnostic challenge. In one of these (patient 8), cross sectional echocardiographic findings were suggestive of total anomalous pulmonary venous drainage; only at operation was hypoplastic left heart syndrome diagnosed, the pulmonary veins draining normally. In another (neonate 3), despite a hypoplastic aortic root, all other chamber dimensions fell in the range of values found in patients with right ventricular overload. A comparison of the anteroposterior and lateral left ventricular cavity dimensions in the short axis projection did not show any difference in the amount of geometric distortion between patients with hypoplastic left heart syndrome and those with other causes of right ventricular overload.

We conclude that no single echocardiographic feature was diagnostic of hypoplastic left heart syndrome. Furthermore, the presence of associated lesions that tend to cause right ventricular overload in their own right may add to the diagnostic difficulty.
A positive diagnosis of hypoplastic left heart syndrome can be made with a high degree of accuracy if it is based on a constellation of echocardiographic features which include (a) the size of the mitral valve annulus and mitral valve mobility, $(b)$ the size of the aortic root and aortic valve mobility, and $(c)$ the size and function of the left ventricle.

1 Freedom RM. Atresia or hypoplasia of the left atrioventricular and/or ventriculo-arterial junction. In: Anderson RH, Shinebourne EA, MacCartney FJ, Tynan M, eds. Paediatric cardiology. London: Churchill LivingM, eds. Paediatric card

2 Cabrera A, Irurita M, Maiz E, Aguirre J, Pastor E. Left ventricular hypoplasia: echocardiographic and anatomical study of 13 cases. Rev Esp Cardiol 1983;36:153-8.

3 Farooki ZQ, Henry JG, Green EW. Echocardiographic spectrum of the hypoplastic left heart syndrome. A clinicopathologic correlation in 19 newborns. Am J Cardiol 1976;38:337-43.

4 Lundstrom NR. Ultrasound studies of the mitral valve region in young infants with mitral atresia, mitral stenosis, hypoplasia of the left ventricle, and cor triatriatum. Circulation 1972;45:324-34.

5 Meyer RA, Kaplan S. Echocardiography in the diagnosis of hypoplasia of the left or right ventricles in the neonate. Circulation 1972;46:55-64.

6 Chesler E, Joffe HS, Vecht R, Beck W, Schrire V. Ultrasound cardiography in single ventricle and the Ultrasound cardiography in single ventricle and the
hypoplastic left and right heart syndromes. Circulation hypoplastic left

7 Bass JL, Ben-Shachar G, Edwards JE. Comparison of $\mathbf{M}$ mode echocardiography with pathologic findings in the hypoplastic left heart syndrome. Am J Cardiol 1979; 45:79-86.

8 Couitz W, Rao PS, Strong WR, Reyes L. Echocardiographic assessment of the aortic root in syndromes with left ventricular hypoplasia. Pediatr Cardiol 1982;2:19-23.

9 Mortera C, Leon G. Detection of persistent ductus in hypoplastic left heart syndrome by contrast echocardiography. Br Heart $J$ 1980;44:596-8

10 Mickell JJ, Mathews RA, Anderson RH, et al. The anatomical heterogeneity of hearts lacking a patent communication between the left atrium and the ventricular mass ('mitral atresia') in presence of a patent aortic valve. Eur Heart $J 1983 ; 4: 477-86$.

11 Tajik AJ, Seward JB, Hagler DJ; Mair DD, Lie JD. Two dimensional real time ultrasonic imaging of the heart and dimensional real time ultrasonic imaging of the

12 Foale R, Nihoyannopoulos P, McKenna W, et al. Echocardiographic measurement of the normal adult right cardiographic measurement of the
ventricle. Br Heart $J 1986 ; 56: 33-44$.

13 Nihoyannopoulos P, Karas S, Sapsford RN, Halliday-Smith $\mathrm{KA}$, Foale RA. Accuracy of two-dimensional echocardiography in the diagnosis of aortic arch obstruction. $J$ Am Coll Cardiol 1987;10:1072-7.

14 Armitage P, Berry G. Statistical methods in medical research. Oxford: Blackwell Scientific Publications, 1987:386-99.

15 Healy MJR. Generalized linear interactive modelling - an introduction. New York: Oxford University Press, 1988.

16 Lachenbruch P, Mickey RM. Estimation of error rates in discriminant analysis. Technometrics 1968;10:1-11.

17 Plomteux G. Multivariate analysis of an enzymatic profile for the differential diagnosis of viral hepatitis. Clin Chem 1980;26:1897-9.

18 Lange LW, Sahn DJ, Allen HD, Ovitt TW, Goldberg SJ. Cross sectional echocardiography in hypoplastic left ventricle: echocardiographic-angiographic-anatomic correlations. Pediat Cardiol 1980;1:287-99. 